УдК 556.06

\title{
ПРОГНОЗНЫЕ ОЦЕНКИ МАКСИМАЛЬНЫХ РАСХОДОВ ВОДЫ РЕКИ ДНЕПР В СТВОРЕ ГОРОДА РЕЧИЦЫ
}

\author{
А. А. Волчек', С. И. Парфомук', С. В. Сидак ${ }^{3}$
}

${ }^{1}$ Д. геогр. н., просрессор, декан фракультета инженерных систем и экологии учреждения образования «Брестский государственный технический университет», Брест, Беларусь, e-mail: volchak@tut.by

${ }^{2}$ К. т. н., доцент, заведующий кафедрой информатики и прикладной математики учреждения образования «Брестский государственный технический университет», Брест, Беларусь, e-mail: parfom@mail.ru

${ }^{3}$ Магистр физ.-мат. н., ассистент кафредры информатики и прикладной математики учреждения образования «Брестский государственный технический университет», Брест, Беларусь, e-mail: harchik-sveta@mail.ru

\section{Ресрерат}

Для получения прогнозных оценок максимального речного стока предложена гибридная модель, основанная на совместном использовании метода эмпирической модовой декомпозиции (EMD) и модели авторегрессии проинтегрированного скользящего среднего (ARIMA). Проведенные анализ и сравнение результатов моделирования максимальных расходов воды р. Днепр в створе г. Речицы позволяют сделать вывод о преимуществе использования гибридной модели EMD-ARIMA перед классической моделью ARIMA. Декомпозиционный подход к прогнозированию рядов максимальных расходов воды весеннего половодья позволяет учесть все его локальные особенности, внутреннюю структуру, а также аномальные выбросы.

Ключевые слова: эмпирическая модовая декомпозиция, преобразование Гильберта-Хуанга, гибридная модель, EMD, ARIMA, прогнозные оценки речного стока.

\section{MAXIMUM WATER RUNOFF FORECAST OF THE DNIEPER RIVER AT THE RECHITSA STATION}

\section{A. A. Volchak, S. I. Parfomuk, S. V. Sidak}

\section{Abstract}

A model based on the combined use of the empirical mode decomposition (EMD) method and the autoregressive integrated moving average (ARIMA) model is proposed to obtain forecast estimates of the maximum river runoff. The analysis and comparison of the results of modeling the maximum water runoff of the Dnieper River at the Rechitsa station allow concluding that the use of the hybrid EMD-ARIMA model is preferable to the classical ARIMA model. The decomposition approach of forecasting maximum water runoff series of the spring flood allows to take into account all its local features, internal structure, as well as abnormal discharges.

Keywords: empirical mode decomposition, Hilbert-Huang transform, hybrid model, EMD, ARIMA, river runoff forecast estimates.

\section{Введение}

Получение прогнозных оценок максимального стока по данным многолетних наблюдений является одной из актуальных проблем гидрологии. Наличие достоверной информации о будущих значениях максимального речного стока является одним из основополагающих факторов эффективного планирования, управления и стабильной работы системы водных ресурсов.

В условиях устойчиво меняющегося климата и объективно возрастающей антропогенной нагрузки на водные ресурсы, прогнозная оценка максимальных расходов воды выступает в качестве важного экономического фактора, который позволяет субъектам хозяйствования выбрать объективную стратегию развития, своевременно принять защитные меры для предотвращения или минимизации ущерба от неблагоприятных и опасных гидрометеорологических явлений. Научная проблема прогнозирования максимальных расходов воды имеет очевидную значимость с точки зрения проектирования, строительства и эксплуатации гидротехнических сооружений, осуществления мероприятий по предотвращению негативного воздействия вод.

На протяжении XX - начала XXI века разрабатывались методики расчета и прогнозирования гидрологических характеристик, основанные на изучении закономерностей многолетних колебаний стока при условии стационарности климата в прошлом и будущем. Однако в настоящее время обоснованность и правильность применения данных методик ставится под сомнение в связи с климатическими изменениями, вызванными процессами потепления климата. Происходящие изменения климата уже привели к изменениям максимального стока рек. Согласно ряду исследований на всех крупных реках Беларуси произошло существенное уменьшение максимальных расходов воды, а также значительно уменьшилась повторяемость наибольших расходов воды малой обеспеченности [1]. Предполагается, что основной причиной изменения максимального стока на территории Беларуси стали смягчение климата в зимний период и повышение частоты зимних оттепелей, в результате чего часть весеннего стока переходит в минимальный зимний сток [2, 3, 4].

Повышение требований к экономической эфффективности и безопасности эксплуатации водных систем приводит к необходимости совершенствования существующих методов долгосрочного прогнозирования максимального речного стока, повышения его точности и заблаговременности.

К настоящему времени разработано большое количество математических моделей и методов анализа и прогнозирования гидрологических временных рядов $[5,6]$.

К наиболее часто применяемым относятся модели, использующие: 1) корреляционный и регрессионный анализы, классификационно-регрессионные деревья; 2) прогнозную экстраполяцию; 3) спектральный анализ, вейвлет-анализ; 4) цепи Маркова; 5) теорию распознавания образов; 6) нейронные сети, генетические алгоритмы.

На сегодняшний день в гидрологии зачастую для оценки риска весенних половодий используют методы, основанные на построении статистических распределений максимальных расходов воды весеннего половодья по имеющимся рядам наблюдений за стоком и дальнейшей 
экстраполяции этих распределений в область малых вероятностей. Использование такого подхода подразумевает выполнение условия однородности и стационарности рядов наблюдения за стоком.

Одной из наиболее распространенных классических статистических моделей прогнозирования является модель ARIMA, предложенная Боксом и Дженкинсом $[7,8]$. Эта модель эффеккивно применяется в таких отраслях, как гидрология, экономика, окружающая среда и политика. Однако гидрологические процессы являются сложными, отражающими взаимодействие большого числа климатообразующих лями. Построенная в результате применения традиционных методов математическая модель гидрологического процесса, как единого и неделимого, является практически нереализуемой и малопригодной для использования в задачах прогноза.

В последнее время в зарубежной литературе появилось много работ $[9,10,11]$, посвященных гибридным декомпозиционным моделям и методам прогнозирования, полученным путем объединения двух и более методов с целью получения лучших характеристик комбинированной гидрологической модели и возможности использования их для прогнозирования нестационарных гидрологических рядов. Основной целью декомпозиционного подхода к прогнозированию является разделение исходного временного ряда на множество рядов с более простой структурой, рассматриваемых независимо друг от друга.

Целью данной статьи является получение прогнозных оценок максимальных расходов воды весеннего половодья рек Беларуси с использованием гибридной модели, основанной на совместном использовании методов эмпирической модовой декомпозиции и БоксаДженкинса.

\section{Исходная информация}

Объектом исследования выбрана первая по величине и водности река Беларуси - Днепр. Река Днепр протекает по территории трех стран - России, Украины и Беларуси. Общая длина реки составляет 2145 км, из них на территории Беларуси - почти 700 км. Река берет свое начало на Валдайской возвышенности в России и впадает в Черное море. Основные правые притоки на территории Беларуси - Друть и Березина, левый - Сож.

В бассейне Днепра на реках Днепр, Сож и Березина, как правило, половодье проходит одной волной. В период весеннего половодья для большинства рек бассейна Днепра характерно затопление поймы. Наиболее продолжительные затопления пойм весной в бассейне Днепра наблюдались в 1956, 1958, 1962, 1970 и 1979 годах. Последнее значительное половодье было в 1999 г.

Бассейн Днепра имеет важную природную и социальноэкономическую значимость в силу того, что на территории бассейна сосредоточены социально-значимые природные ресурсы (например водные, земельные и лесные ресурсы), а также является ценной ресурсной базой для промышленных предприятий, землепользователей, водопользователей, правительственных структур, органов госконтроля и регулирования). Регион бассейна Днепра на территории Беларуси является развитым как в промышленном, так и в аграрном отношении, в связи с чем влияние ресурсов поверхностных и подземных вод на социальное развитие и основные секторы экономики являются существенным [12].

Все это оправдывает актуальность изучения современных изменений максимального стока рек бассейна Днепра как в связи с его хозяйственным значением, так и со стороны влияния меняющегося климата на характеристики речного стока.

В исследовании использованы данные гидрологических наблюдений за максимальными расходами воды весеннего половодья реки Днепр за период инструментальных наблюдений Государственного учреждения «Республиканский центр по гидрометеорологии, контролю радиоактивного загрязнения и мониторингу окружающей среды» Министерства природных ресурсов и охраны окружающей среды Республики Беларусь. факторов, каждый из которых может быть описан различными моде-

Получение прогнозных оценок изменения максимального стока реки Днепр, обусловленного влиянием климатических факторов, выполнено для створа г. Речицы. Пропуски в рядах данных были восстановлены с помощью компьютерного программного комплекса «Гидролог» [13]. Период исследования составил 141 год (1877-2017 гг.).

\section{Методы исследования}

В данной работе для прогнозирования максимального речного стока предлагается гибридная модель EMD-ARIMA, основанная на совместном использовании метода эмпирической модовой декомпозиции (empirical mode decomposition, EMD) и модели авторегрессии проинтегрированного скользящего среднего (autoregressive integrated moving average, ARIMA) [14]. EMD используется для разложения исходного нестационарного ряда на серию модовых функций (intrinsic mode functions, IMF) и остаток, к каждому из которых может быть применена ARIMA-методология (рисунок 1).

Основными этапами получения прогнозных оценок с помощью гибридной модели EMD-ARIMA являются:

1) последовательные операции по выделению из исходного временного ряда модовых функций, начиная с высокочастотных, и остатка;

2) разработка подходящей модели ARIMA для каждой IMF функции и остатка;

3) проведение общих вычислений для прогнозирования исходного временного ряда на основе прогноза каждой подсерии;

4) сравните производительности гибридной модели EMD-ARIMA со стандартной моделью ARIMA.

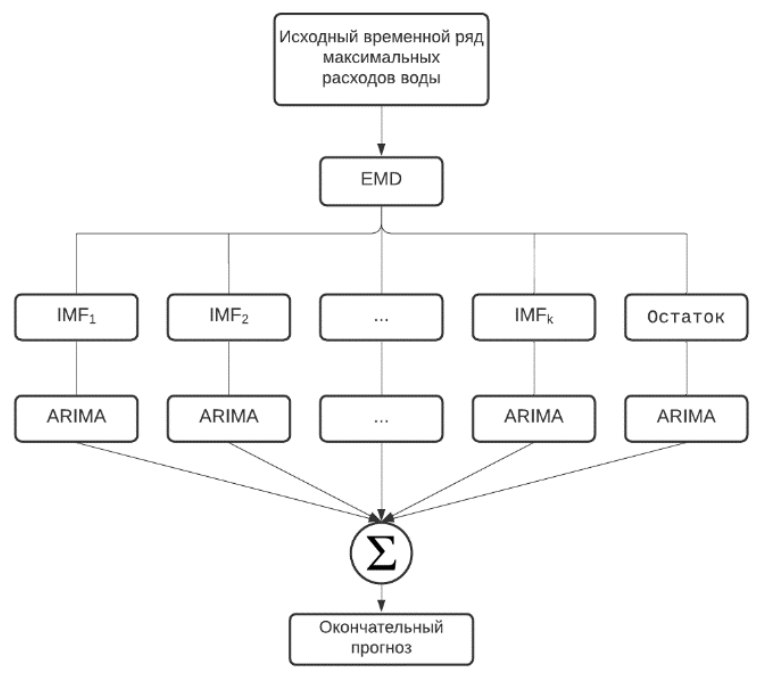

Рисунок 1 - Блок-схема гибридной модели EMD-ARIMA

Ниже описаны оба метода, входящих в основу гибридной модели.

\section{Memod EMD}

Одним из методов, входящих в состав гибридной модели, представлен метод EMD, который является относительно новой формой разложения временных рядов. Особенность метода состоит в том, что временной ряд может не являться линейным или стационарным. Необходимым условием корректного представления нелинейных и нестационарных процессов является возможность формирования адаптивного базиса, функционально зависимого от содержания самих данных.

Метод EMD является важнейшей составляющей преобразования Гильберта-Хуанга (Huang-Hilbert Transform - HНT), широко используемого в различных областях науки и техники, наряду с преобразованием Фурье и вейвлет анализом [15].

Отличие метода EMD от вейвлет-анализа состоит в том, что в процессе EMD производится разложение на серию модовых функций, которые не заданы аналитически и определяются исключительно самой анализируемой последовательностью данных, 
Вестник Брестского государственного технического университета. 2021

причем базисные функции преобразования формируются адаптивно, непосредственно из входных данных. При вейвлет-преобразовании, так же как и при преобразовании Фурье, производится разложение в фиксированном базисе функций. Этот базис должен быть предварительно задан, то есть должна быть выбрана конкретная вейвлетфункция, используемая в процессе преобразования.

Основная идея метода EMD заключается в предположении, что исследуемый процесс представляет собой аддитивную комбинацию различных внутренних колебаний, каждое из которых представляет собой модовую функцию, имеющую экстремумы и нулевые значения $[16,17]$.

Каждая IMF функция должна удовлетворять двум критериям:

1) число экстремумов функций и число нулевых пересечений должны быть равными либо отличаться на единицу;

2) в любой точке функций среднее значение огибающих, интерполирующих локальные максимумы и минимумы, должно быть нулевым.

Пусть $Q(t)$ - многолетний ряд максимальных расходов воды. Тогда основная идея метода EMD состоит в разложении временного ряда $Q(t)$ на IMF-фуннции и остаток $r(t)$. В результате такого разложения ряд $Q(t)$ может быть представлен в виде:

$$
Q(t)=\sum_{j=1}^{k} I M F_{i}(t)+r_{k}(t),
$$

где $r_{k}(t)$ - остаточная составляющая декомпозиции временного ряда, $I M F_{i}(t)$ - i-я внутренняя модовая функция.

Алгоритм процесса EMD состоит из следующих этапов (рисунок 2):

1) выбор ряда $Q(t)$ для процесса просеивания; ввод в рассмотрение трех переменных $i, j$ и $k$, где $i$ - номер вычисляемой IMF, $j$ - номер ее приближения, $k$ - количество IMF (начальные значения переменных: $i=1, j=1, k=0$ );

2) ввод в рассмотрение дополнительного ряда $S_{i}(t)=Q(t)$;

3) идентификация всех локальных экстремумов во временном ряду $Q(t) ;$

4) формирование верхней $p(t)$ и нижней $q(t)$ огибающих, соединив все локальные максимумы и локальные минимумы соответственно;

5) определение средней функции $\phi_{j}(t)=\frac{p(t)+q(t)}{2}$;

6) вычисление первой компоненты отсеивания $\psi_{j}(t)=Q(t)-\phi_{j}(t)$. В случае, если $\psi_{j}(t)$ является IMF функцией, переходим к следующему шагу. В противном случае в качестве значений ряда $Q(t)$ используем $\psi_{j}(t)$, а значение переменной $j$ увеличиваем на $1(j=j+1)$. Для обновленного ряда $Q(t)$ повторяем этапы $3-5$;

7) этот этап состоит из двух шагов. Первый - сохраняем $\psi_{j}(t)$, полученную на предыдущем шаге, в качестве $I M F_{i}(t)$, т. е. $I M F_{i}(t)=\psi_{j}(t)$, значение переменной $k$ увеличиваем на единицу. Второй - по формуле (2) определяем остаток $r_{i}(t)$ :

$$
r_{i}(t)=S_{i}(t)-I M F_{i}(t)
$$

8) в соответствии с характеристиками функции $r_{i}(t)$, полученной на 7 этапе, принимается решение об останове вычислений. Вычисления прекращаются в следующих случаях:

- $r_{i}(t)$ является константой или постоянной функцией, из которой больше не может быть извлечено IMF функций;

- остаток $r_{i}(t)$ на всем интервале исследования становится несущественным по своим значениям по сравнению с исходным рядом;

9) в случае, если критерии останова процесса EMD не выполнены, в качестве значений ряда $Q(t)$ используем $r_{i}(t)$, значение переменной $i$ увеличиваем на $1(i=i+1)$, переменной $j$ присваиваем значение 1. Далее переходим к этапу 2 и продолжаем процесс EMD. Важнейшим этапом реализации метода EMD является процесс построения верхней и нижней огибающих локальных экстремумов. Традиционно для этих целей используют кубические сплайны и В-сплайны. Однако при интерполяции этими видами сплайнов может возникнуть такое явление, как краевой эффект (рисунок 3). Причиной концевых искажений модовых функций является непредсказуемость аппроксимации по экстремумам верхней и нижней огибающих на концевых участках мод.

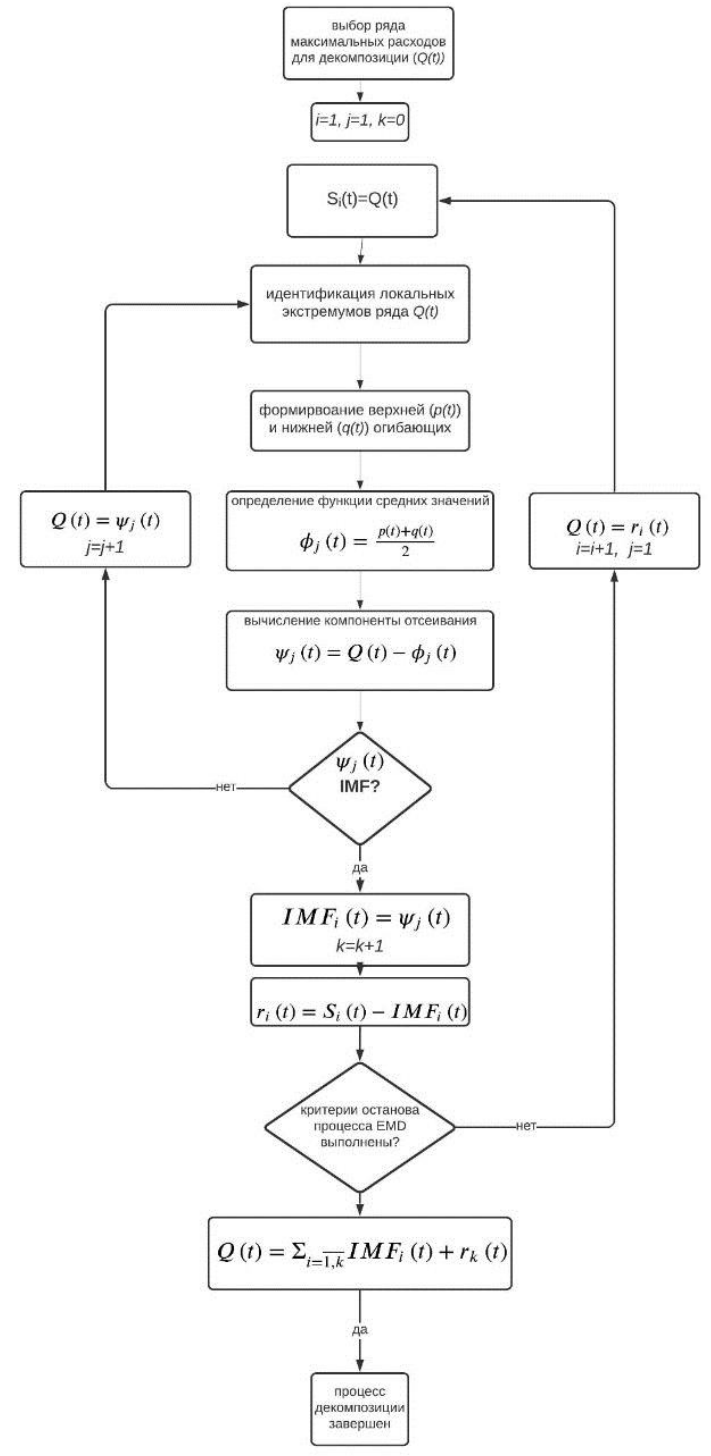

Рисунок 2 - Блок-схема процесса EMD

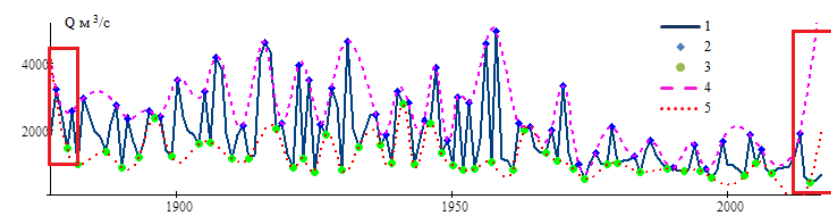

1 - наблюдаемые данные, 2 - локальные максимумы, 3 - локальные минимумы,

4 - верхняя огибающая, 5 - нижняя огибающая

Рисунок 3 - Краевой эффеект при интерполяции кубическими сплайнами

В силу того, что процесс построения модовых функций является последовательным вычитанием текущей вычисляемой моды из предыдущих входных данных максимальных расходов воды, ошибки аппроксимации огибающих на концевых точках приводят к искажениям вычисляемых мод и рекурсивному накоплению ошибок вычисления модовых функций. Это значительно влияет на результаты разложения EMD. 
Для ослабления краевых эффектов на концах ряда в данной работе предлагается использование истокообразной аппроксимации при построении огибающих экстремумов [18, с. 101]. Применение данного подхода для снижения влияния концевых эффеектов на процесс EMD позволяет стандартизовать процесс уменьшения краевых искажений модовых функций.

\section{Memod ARIMA}

В общем виде модель $\operatorname{ARIMA}(p, d, q)$ для нестационарного ряда $y_{t}$ выражается формулой

$$
\Delta^{d} y_{t}=c+\sum_{i=1}^{p} \alpha_{i} \Delta^{d} y_{t-i}+\sum_{j=1}^{q} b_{j} \varepsilon_{t-j}+\varepsilon_{t}
$$

где $p$ - параметр авторегрессии, $d$ - порядок операции взятия разностей, $q$ - порядок скользящего среднего, $c$ - константа, $\alpha_{i}, b_{j}$ коэфффициенты модели, $\Delta^{d} y_{t}$ - оператор разности $d$-го порядка ( $\Delta y_{t}=y_{t}-y_{t-1}-$ разности первого порядка), $\varepsilon_{t}-$ «белый шум» [19].

B основе прогнозирования с использованием ARIMA-моделей лежит методология Бокса-Дженкинса, которая содержит три этапа:

1) идентификация модели (определение параметров $d, p, q)$;

2) оценивание и проверка адекватности модели;

3) прогнозирование.

На первом этапе модели необходимо проанализировать ряды на стационарность и подобрать ARIMA-модель для дальнейшего оценивания. На втором этапе проводится оценка параметров ARIMAмоделей методом максимального правдоподобия и проверка адекватности полученных ARIMA-моделей. Для их сравнения используют несколько критериев: оценки коэффициентов модели должны быть статистически значимыми, остатки модели должны обладать свойствами белого шума. Если несколько ARIMA моделей оказываются адекватными, необходимо выбрать модель с наименьшим количеством параметров и наилучшими статистическими характеристиками качества подгонки модели. В данной работе для этого использован информационный критерий Акаике $(A I C)$ :

$$
\begin{aligned}
& A I C(p, q)=\ln \sigma^{2}+\frac{2 k}{N}, \\
& \sigma^{2}=\frac{R S S}{N-p-q}, k=p+q,
\end{aligned}
$$

где $p, q$ - параметры ARIMA модели, $N$ - число наблюдений, $R S S$ остаточная сумма квадратов.

\section{Оценка эффективности модели}

Для оценки эффективности используемой в работе гибридной модели EMD-ARIMA по формулам (5)-(8) получены значения среднеквадратической ошибки (RMSE), средней абсолютной ошибки (MAE), средней абсолютной ошибки в процентах (MAPE), коэффициента корреляции (R) для обучающей и тестовой выборок.

$$
\begin{aligned}
& \text { RMSE }=\sqrt{\frac{1}{N} \sum_{i=1}^{N}\left(Q^{o}{ }_{i}-Q^{m}{ }_{i}\right)^{2},} \\
& \text { MAE }=\frac{1}{N} \sum_{i=1}^{N}\left|Q^{o}{ }_{i}-Q^{m}{ }_{i}\right|, \\
& \text { MAPE }=\frac{1}{N} \sum_{i=1}^{N}\left|\frac{Q^{o}{ }_{i}-Q^{m}{ }_{i}}{Q^{o}}\right| \cdot 100 \%, \\
& \mathrm{R}=\frac{\sum_{i=1}^{N}\left(Q^{m}{ }_{i}-Q^{m}\right)\left(Q^{o}{ }_{i}-Q^{o}\right)}{\sqrt{\sum_{i=1}^{N}\left(Q^{m}{ }_{i}-Q^{m}\right)^{2}} \sqrt{\sum_{i=1}^{N}\left(Q^{o}{ }_{i}-\bar{Q}^{o}\right)^{2}}},
\end{aligned}
$$

где $N$ - объем выборки, $Q^{\circ}{ }_{i}$ - наблюдаемые данные, $Q^{m}{ }_{i}-$ смоделированные данные, $\overline{Q^{\circ}}$ - среднее значение наблюдаемых данных, $\overline{Q^{m}}$ - среднее значение смоделированных данных.

\section{Результаты и их обсуждение}

На рисунке 4 изображен хронологический график многолетних колебаний максимальных расходов стока реки Днепр в створе г. Речицы за период с 1877 по 2017 гг. В данном исследовании данные максимальных расходов воды с 1877 по 1997 гг. использованы для обучения как ARIMA, так и EMD-ARIMA модели. Затем полученная модель применена к тестовым данным (период с 1998 по 2017 гг.).

На рисунке 5 продемонстрирован первый этап процесса EMD извлечение локальных экстремумов ряда максимальных расходов речного стока.

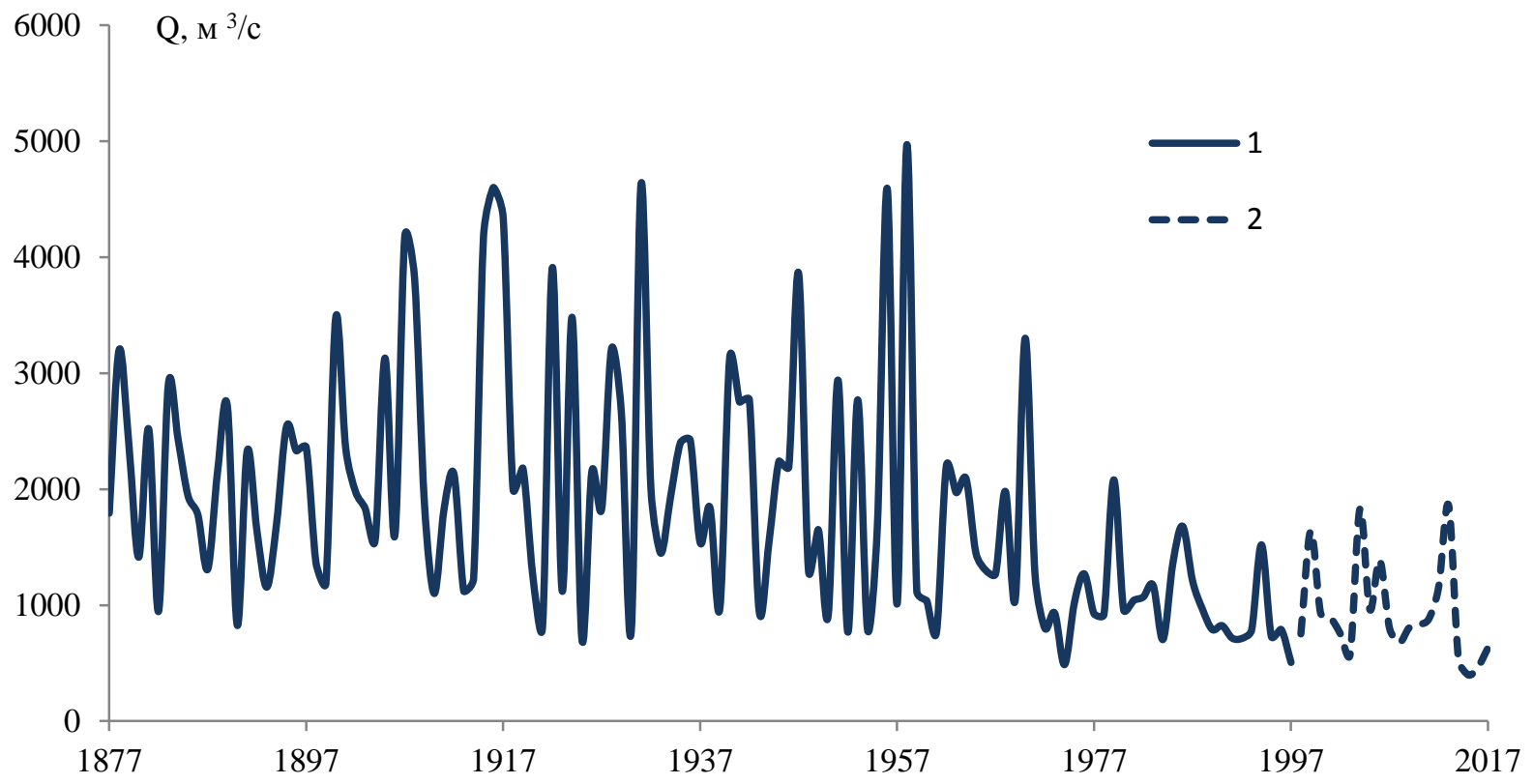

1 - обучающая выборка, 2 - тестовая выборка

Рисунок 4 - Многолетние изменения максимального стока реки Днепр в створе г. Речицы за период 1877-2017 гг 


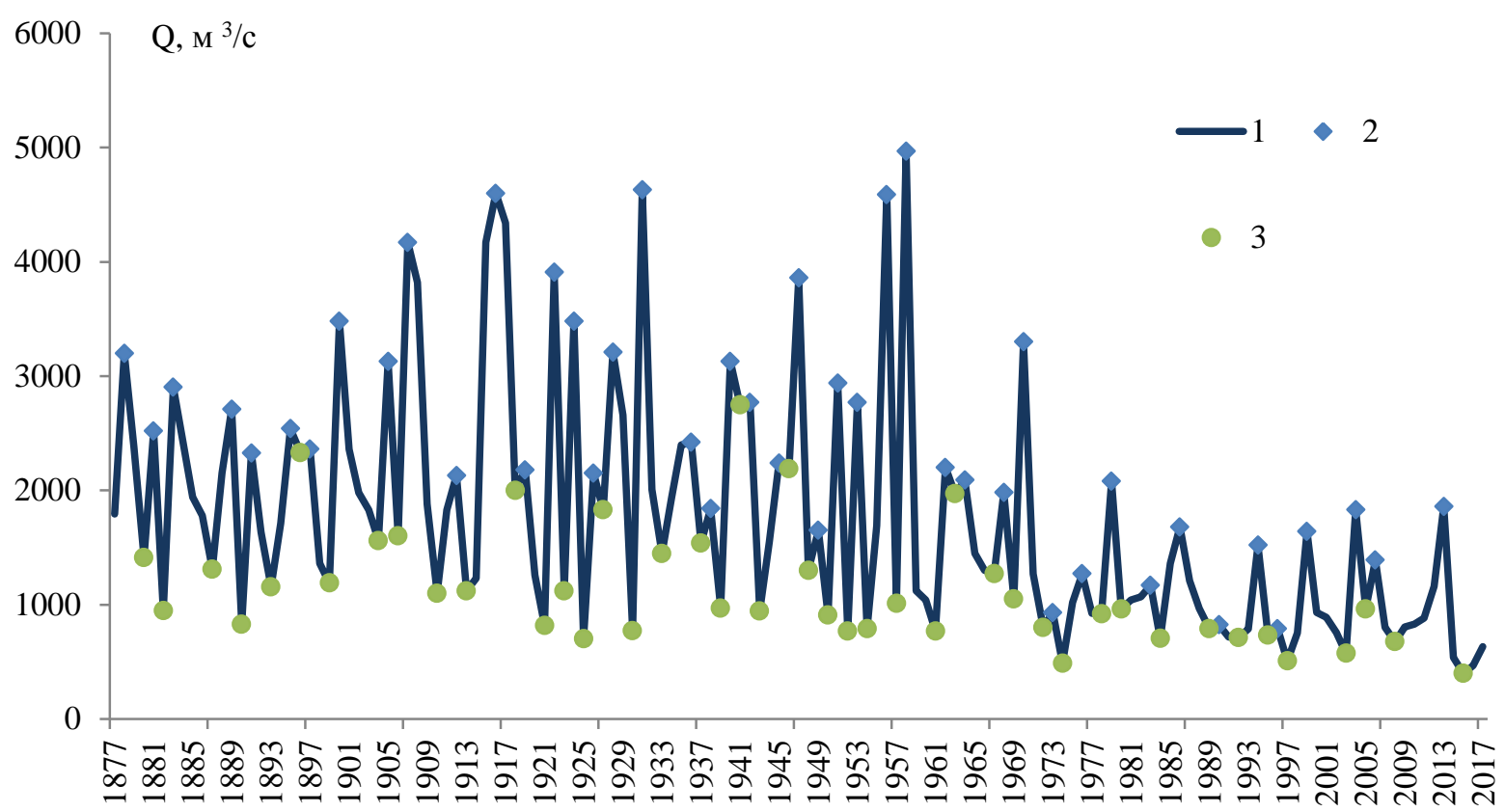

1 - наблюдаемые данные, 2 - локальные максимумы, 3 - локальные минимумы

Рисунок 5 - Извлечение локальных экстремумов в исходном ряду данных

На рисунке 6 приведен пример построения IMF1. В этом примере извлеченная функция не удовлетворяет условиям IMF (количество экстремумов и нулевых пересечений отличается более чем на единицу) и, следовательно, в дальнейшем необходимо выполнение процедуры просеивания.
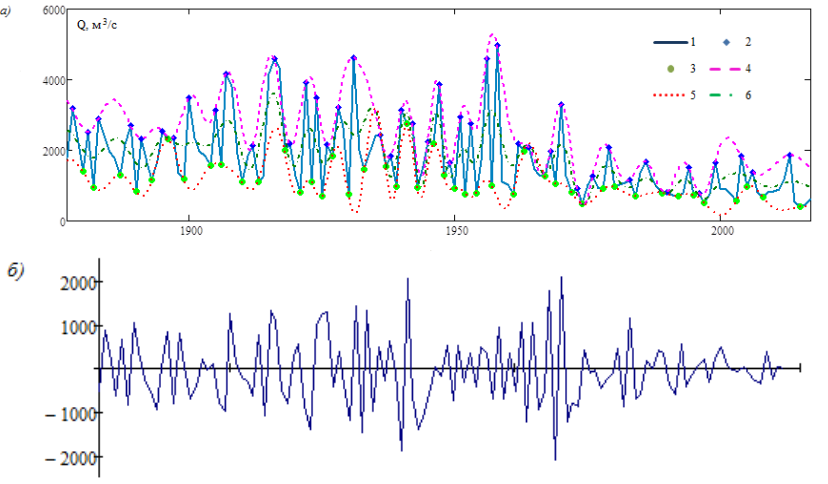

\footnotetext{
а) 1 - наблюдаемые данные, 2 - локальные максимумы, 3 - локальные минимумы,

4 - верхняя огибающая, 5 - нижняя огибающая, 6 - функция средних значений огибающих;

б) первая компонента отсеивания

Рисунок 6 - Построение первого приближения к IMF1
}

Результат процесса эмпирической модовой декомпозиции ряда максимальных расходов воды представлен на рисунке 7.

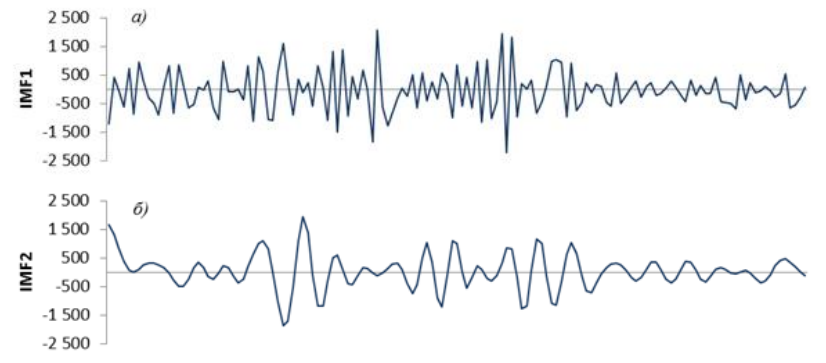

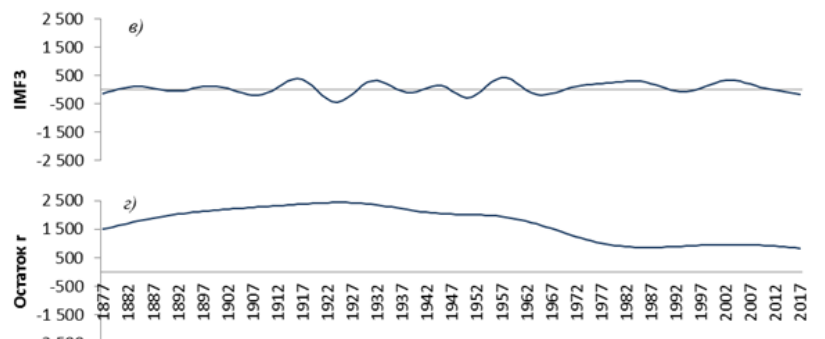

a - IMF1, б - IMF2, в - IMF3, г - остаток r

Рисунок 7 - Разложение ряда максимальных расходов воды на функции IMF и остаток r

Некоторые сложности при использовании EMD на практике связаны с появлением случаев коррелирующих между собой IMF (эффект микширования мод) [20]. Для подавления этого эффректа необходимо вычислить парные коэффицциенты линейной корреляции и суммировать функции, имеющие тесную корреляционную связь.

Таблица 1 - Парные коэффициенты корреляции IMF функций разложения ряда максимальных расходов воды

\begin{tabular}{|l|c|c|c|}
\hline & IMF1 & IMF2 & IMF3 \\
\hline IMF1 & 1 & - & - \\
\hline IMF2 & $-0,072$ & 1 & - \\
\hline IMF3 & $-0,041$ & $-0,003$ & 1 \\
\hline
\end{tabular}

Все коэффицциенты парной корреляции из таблицы 1 незначимы, поэтому для данного исследования в процедуре суммирования мод нет необходимости.

Для обоснованного вывода о стационарности полученных IMF и остатка $r$ построим для них автокорреляционную (АКФ) и частную автокорреляционную функции (ЧАКФ). Для этого определим первые 25 значений коэфффициентов автокорреляции. На рисунке 8 приведены коррелограммы АКФ и ЧАКФ.

Рисунок 8 показывает, что компоненты IMF и $r$ не являются ни чистыми моделями AR, ни MA, а моделями ARMA и ARIMA. Для прогнозирования каждой IMF и остатка из всего портфеля моделей выбраны те, которые имеют минимальные значения AIC. 


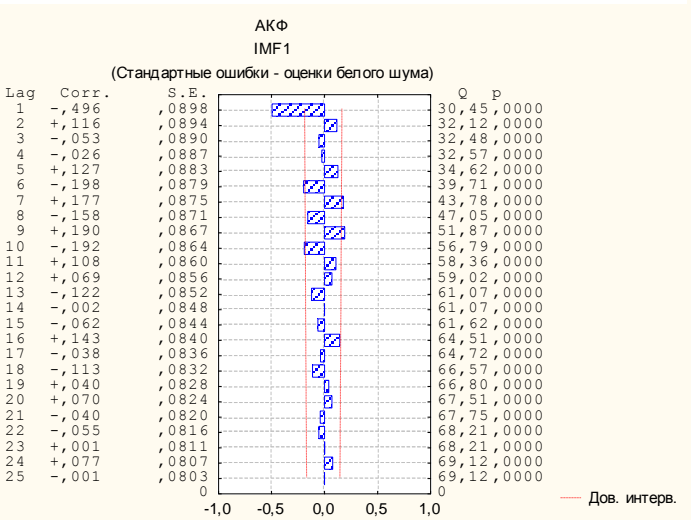

a)

AKФ

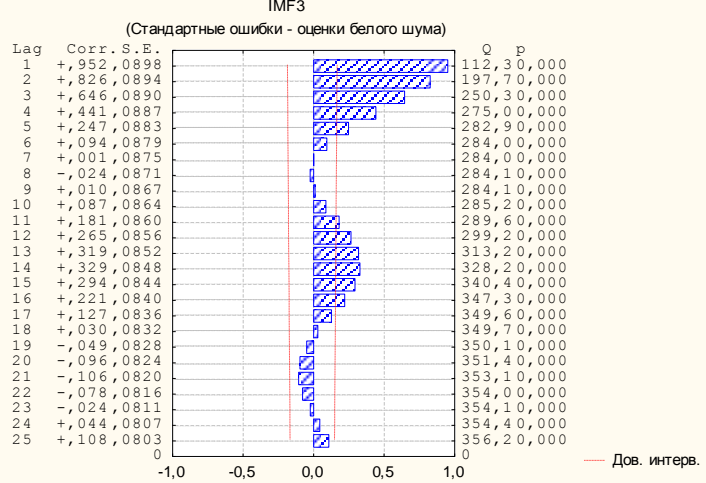

B)

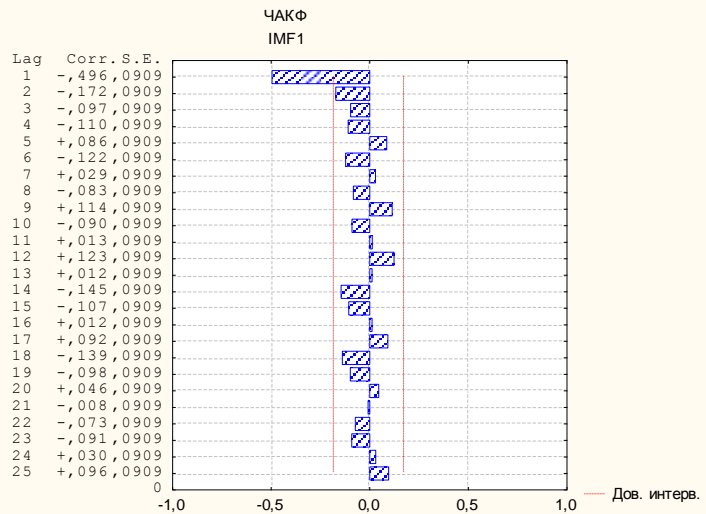

д)

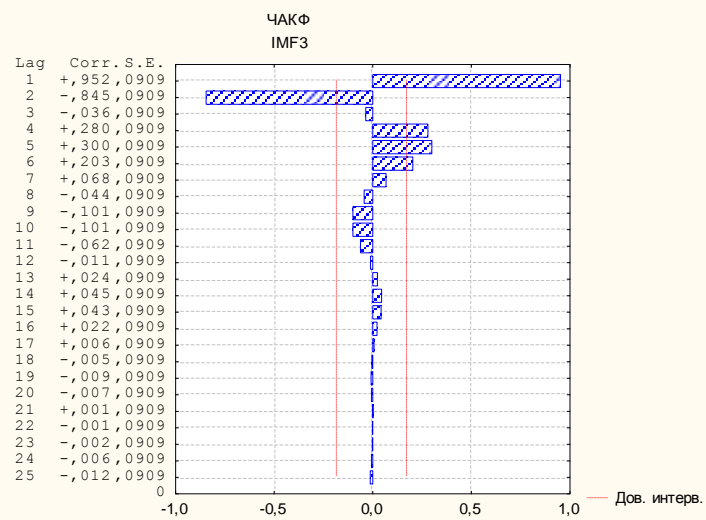

ж)

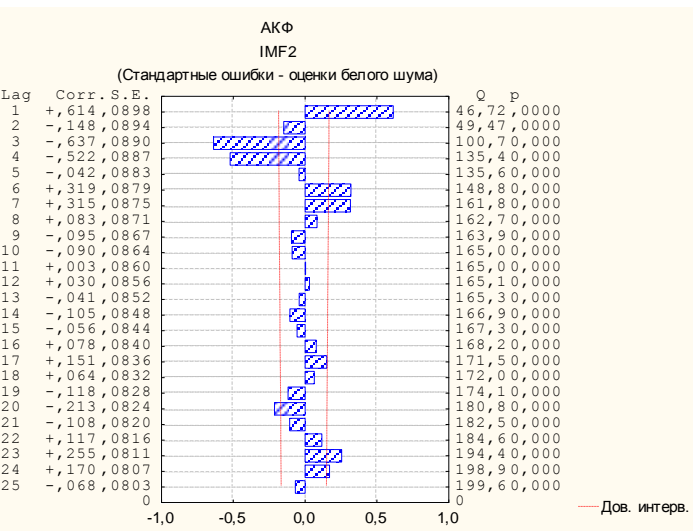

б)

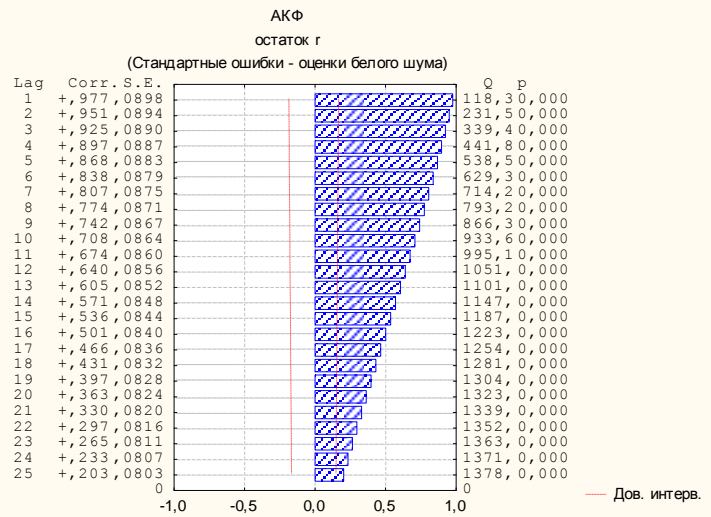

Г)

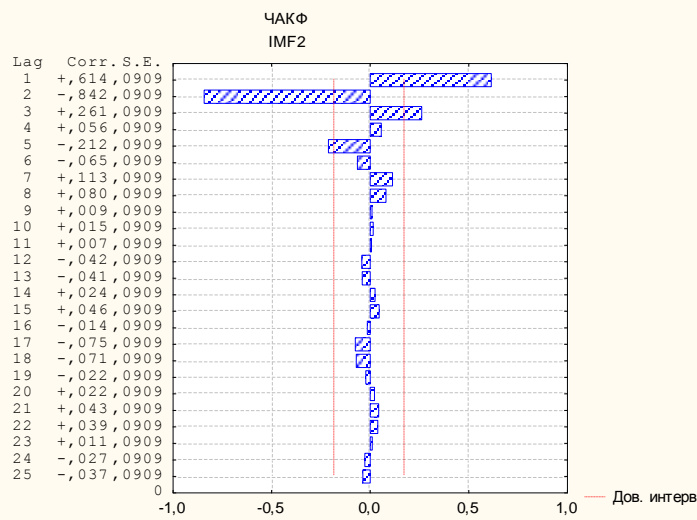

e)

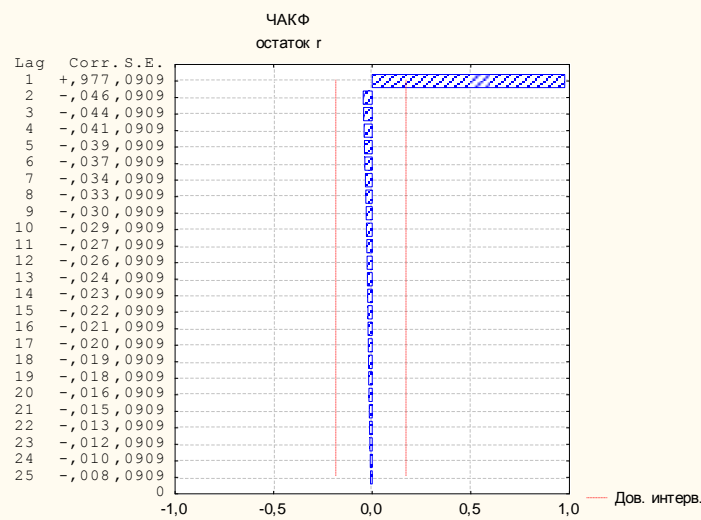

3)

Рисунок 8 - Графики автокорреляционной и частной автокорреляционной функций для полученных в процессе EMD IMF:

AKФ - a) IMF1, б) IMF2, в) IMF1, г) остаток r;

ЧАКФ - д) IMF1, е) IMF2, ж) IMF1, з) остаток $r$ 
Вестник Брестского государственного технического университета. 2021

\begin{tabular}{|c|c|c|c|}
\hline Модель & IMF & ARIMA & AIC \\
\hline ARIMA & \multicolumn{2}{|c|}{$\operatorname{ARIMA}(7,1,0)$} & 13,24 \\
\hline \multirow{4}{*}{$\begin{array}{c}\text { Гибридная } \\
\text { модель } \\
\text { EMD-ARIMA }\end{array}$} & IMF1 & ARIMA $(3,0,5)$ & 13,15 \\
\hline & IMF2 & $\operatorname{ARIMA}(7,0,4)$ & 11,20 \\
\hline & IMF3 & ARIMA $(1,4,1)$ & 3,15 \\
\hline & Остаток & ARIMA $(4,4,2)$ & $-0,71$ \\
\hline
\end{tabular}

Параметры оценки эффрективности моделей ARIMA и EMD-ARIMA для обучающей и тестовой выборок представлены в таблице 3 .

Как видно из таблицы 3 и рисунка 9 модельные данные, полученные с использованием гибридной модели EMD-ARIMA, лучше согласуются с наблюдаемыми данными по сравнению с классической моделью ARIMA. Метод EMD-ARIMA даёт меньшие значения ошибок как на наборе обучающих данных, так и на тестовой выборке.

На рисунке 10 представлены прогнозные оценки максимальных расходов воды р. Днепр в створе г. Речицы на период 2018-2027 гг., полученные с использованием лучшей из двух рассматриваемых в работе моделей - гибридной модели EMD-ARIMA.

Таблица 3 - Значения показателей эффрективности моделей прогнозирования

\begin{tabular}{|c|c|c|c|c|c|c|c|c|}
\hline & \multicolumn{3}{|c|}{ Обучающая выборка } & \multicolumn{3}{c|}{ Тестовая выборка } \\
\hline $\begin{array}{c}\text { Mo- } \\
\text { дель }\end{array}$ & $\begin{array}{c}\text { RMSE, } \\
\mathrm{M}^{3} / \mathrm{c}\end{array}$ & $\begin{array}{c}\mathrm{MAE}, \\
\mathrm{M}^{3} / \mathrm{c}\end{array}$ & $\begin{array}{c}\text { MAPE, } \\
\%\end{array}$ & $\mathrm{R}$ & $\begin{array}{c}\text { RMSE, } \\
\mathrm{M}^{3} / \mathrm{c}\end{array}$ & $\begin{array}{c}\text { MAE, } \\
\mathrm{M}^{3} / \mathrm{c}\end{array}$ & $\begin{array}{c}\text { MAPE, } \\
\%\end{array}$ & $\mathrm{R}$ \\
\hline ARIMA & 684,46 & 511,70 & 30 & 0,76 & 373,15 & 273,85 & 30 & 0,66 \\
\hline $\begin{array}{c}\text { EMD- } \\
\text { ARIMA }\end{array}$ & 630,90 & 467,79 & 29 & 0,80 & 278,45 & 217,31 & 24 & 0,76 \\
\hline
\end{tabular}

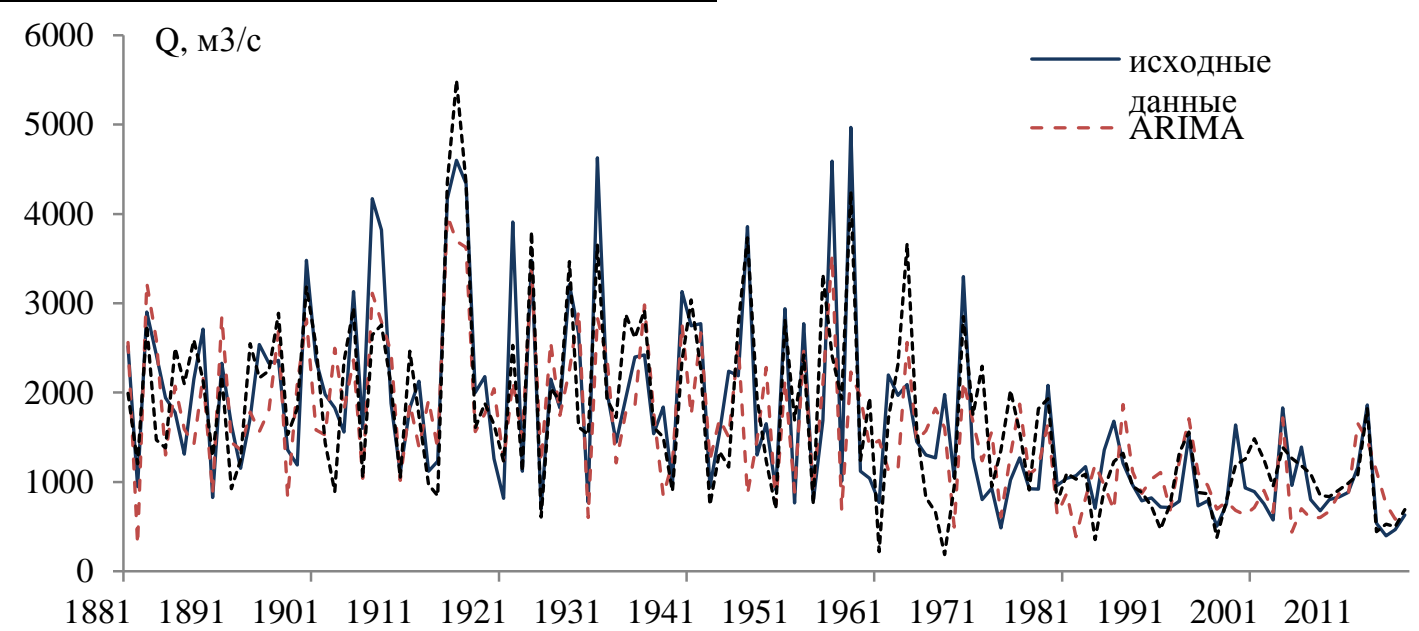

Рисунок 9 - Сопоставление фактических и смоделированных значений максимальных расходов воды р. Днепр в створе г. Речица

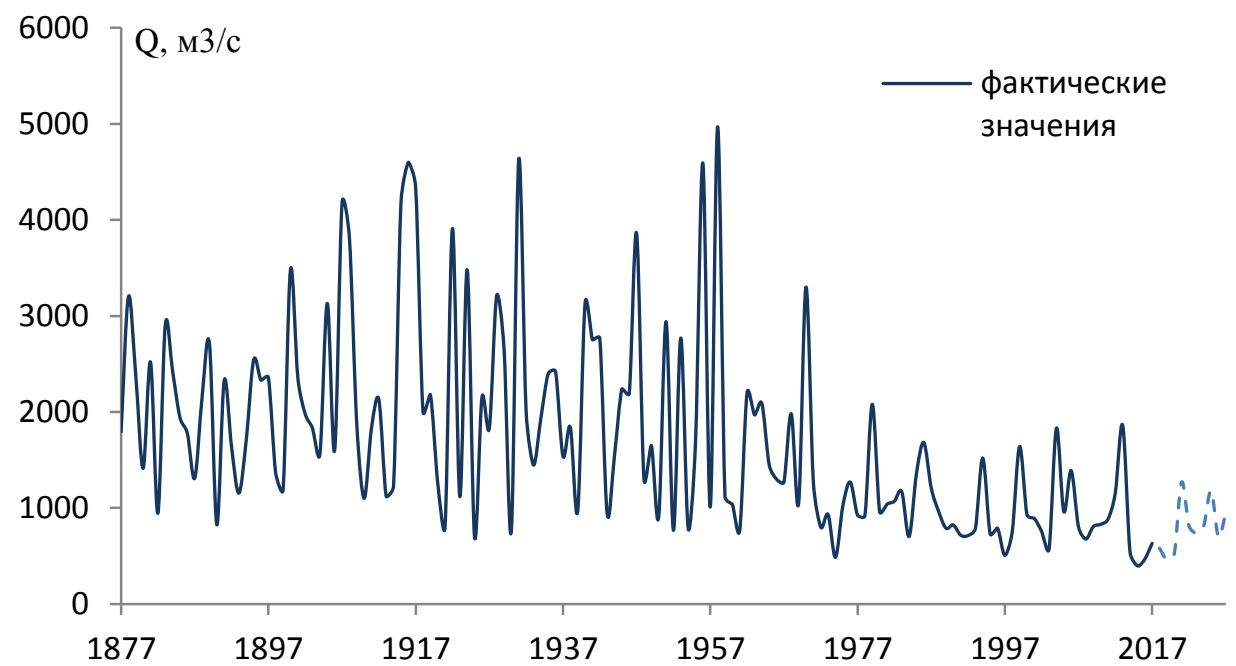

Рисунок 10 - Прогнозные оценки максимальных расходов воды р. Днепр в створе г. Речица на период 2018-2027 гг.

\section{Заключение}

В данном исследовании для прогнозирования нестационарного временного ряда максимальных расходов речного стока предлагается гибридная модель EMD-ARIMA. Основная идея использования предложенной модели состоит в использовании метода EMD для разложения ряда максимальных расходов речного стока на отдельные IMF и остаток и подборе подходящих моделей ARIMA для полученных серий IMF. Окончательный прогноз получается путем объединения результатов прогнозов различными моделями ARIMA каждой серии. Метод является сравнительно простым в вычислительном плане и не требует выполнения условия стационарности гидрологических рядов.

Анализ показателей эффективности при моделировании максимальных расходов воды позволяет сделать вывод о преимуществе предложенной гибридной модели EMD-ARIMA перед классической моделью ARIMA. Полученные в работе результаты показали, что предложенная гибридная модель способна предсказывать значения максимальных расходов речного стока с высокой точностью.

Работа выполнена при поддержке БРФФИ (грант № Х20М064) 


\section{Список цитированных источников}

1. Волчек, А.А. Оценка современных изменений максимального стока рек Беларуси / А. А. Волчек, Ан. А. Волчек, С. В. Сидак // География. - Минск, 2020. - № 4(167). - С. 26-33.

2. Волчек, Ан. А. Затопления на территории Беларуси / Ан. А. Волчек // Вестник Брестского государственного технического университета. 2017. - № 2: Водохозяйственное строительство, теплоэнергетика и геоэкология. - С. 39-53.

3. Логинов, В. Ф. Весенние половодья на реках Беларуси: пространственно-временные колебания и прогноз / В. Ф. Логинов, А. А. Волчек, Ан. А. Волчек - Минск : Беларуская навука, 2014. - 244 с.

4. Волчек, А. А. Особенности внутригодового распределения стока рек бассейна Припяти / А.А. Волчек, С.В. Сидак // Вестник БрГТУ. 2020. - № 2(120): Водохозяйственное строительство, теплоэнергетика и геоэкология. - С. 2-9.

5. Георгиевский, Ю. М. Гидрологические прогнозы / Ю. М. Георгиевский, С. В. Шаночкин. - С.-Пб.: РГГМУ, 2007.

6. Кучмент, Л. С. Речной сток (генезис, моделирование, предвычисление) / Л. С. Кучмент. - М., 2008.

7. G.E.P. Box and G.M. Jenkins, Time Series Analysis Forecasting and Control, Holden-Day, San Francisco, 1970.

8. Balyani Y., Niya G.F., and Bayaat A., 2014. A study and prediction of annual temperature in Shiraz using ARIMA model. J. Geographic Space, 12(38), 127-144.

9. Wang W, Van Gelder P, Vrijling JK, Ma J (2006) Forecasting daily streamflow using hybrid ANN models. J Hydrol 324: 383-399

10. Jain A, Kumar AM (2007) Hybrid neural network models for hydrologic time series forecasting. Appl Soft Comput 7: 585-592

11. Sahoo GB, Schladow SG, Reuter JE (2009) Forecasting stream water temperature using regression analysis, artificial neural network, and chaotic non-linear dynamic models. J Hydrol 378: 325-342.

12. План управления речным бассейном Днепра. - Минск : ЦНИИКИВР 2019 г. -82 c.

13. Волчек, А. А. Автоматизация гидрологических расчетов / А. А. Волчек // Водохозяйственное строительство и охрана окружающей среды: труды Международной научно-практической конференции по проблемам водохозяйственного, промышленного и гражданского строительства и экономико-социальных преобразований в условиях рыночных отношений. / Брест. политехн. институт. - Биберах - Брест - Ноттингем, 1998. - С. 55-59.

14. Z. Wang, J. Qiu, and F. Li, "Hybrid Models Combining EMD/ EEMD and ARIMA for Long-Term Streamfow Forecasting," Water, vol. 10, no. 7, p. 853, 2018.

15. Norden E. Huang, Samuel S.P. Shen. The Hilbert-Huang transform and its applications // World Scientific Publishing Co. Pte. Ltd. 2005. - $311 \mathrm{c}$.

16. Peel MC, G.G.S. Pegram, and T.A. McMahon. 2007: Empirical Mode Decomposition: Improvement and application. In International Congress on Modeling and Simulation, edited by Oxley, L. and D. Kulasiri. Modelling and Simulation Society of Australia and New Zealand, December 2007, 2996-3002.

17. Huang N. E., etc. The Empirical Mode Decomposition and The Hilbert Spectrum for Non-linear and Non-stationary Time Series Analysis// Proc. Royal Soc. London, Vol. 454, p. 903-995, 1998.

18. Долгаль, А. С. Применение эмпирической модовой декомпозиции при обработке геофизических данных / А. С. Долгаль, Л. А. Христенко // Изв. Томского политехнич. ун-та. Инжиниринг ресурсов. 2017. - T. 328. - № 1. - С. 100-108.

19. Айвазян, С. А. Методы эконометрики: учебник / С. А. Айвазян. М. : Инфрра-М, 2014. - 512 c.

20. Христенко, Л. А. Эмпирическая модовая декомпозиция при интерпретации данных электроразведки / Л. А. Христенко // Геология и полезные ископаемые Западного Урала. - Пермь, 2019. № 2(39). - C. 222-226.

\section{References}

1. Volchek, A. A. Ocenka sovremennyh izmenenij maksimal'nogo stoka rek Belarusi / A. A. Volchek, An. A. Volchek, S. V. Sidak // Geografiya. - Minsk, 2020. - № 4(167). - S. 26-33.

2. Volchek, An. A. Zatopleniya na territorii Belarusi / An. A. Volchek // Vestnik Brestskogo gosudarstvennogo tekhnicheskogo universiteta. 2017. - № 2: Vodohozyajstvennoe stroitel'stvo, teploenergetika i geoekologiya. - S. 39-53.

3. Loginov, V. F. Vesennie polovod'ya na rekah Belarusi: prostranstvenno-vremennye kolebaniya i prognoz / V. F. Loginov, A. A. Volchek, An. A. Volchek - Minsk : Belaruskaya navuka, 2014. - $244 \mathrm{~s}$.

4. Volchek, A. A. Osobennosti vnutrigodovogo raspredeleniya stoka rek bassejna Pripyati / A.A. Volchek, S.V. Sidak // Vestnik BrGTU. 2020. - № 2(120) : Vodohozyajstvennoe stroitel'stvo, teploenergetika i geoekologiya. - S. 2-9.

5. Georgievskij, YU. M. Gidrologicheskie prognozy / YU. M. Georgievskij, S. V. SHanochkin. - S.-Pb.: RGGMU, 2007.

6. Kuchment, L. S. Rechnoj stok (genezis, modelirovanie, predvy-chislenie) / L. S. Kuchment. - M., 2008.

7. G.E.P. Box and G.M. Jenkins, Time Series Analysis Forecasting and Control, Holden-Day, San Francisco, 1970.

8. Balyani Y., Niya G.F., and Bayaat A., 2014. A study and prediction of annual temperature in Shiraz using ARIMA model. J. Geographic Space, 12(38), 127-144.

9. Wang W, Van Gelder P, Vrijling JK, Ma J (2006) Forecasting daily streamflow using hybrid ANN models. J Hydrol 324: 383-399

10. Jain A, Kumar AM (2007) Hybrid neural network models for hydrologic time series forecasting. Appl Soft Comput 7: 585-592

11. Sahoo GB, Schladow SG, Reuter JE (2009) Forecasting stream water temperature using regression analysis, artificial neural network, and chaotic non-linear dynamic models. J Hydrol 378: 325-342.

12. Plan upravleniya rechnym bassejnom Dnepra. - Minsk : CNIIKIVR, 2019 g. -82 c.

13. Volchek, A. A. Avtomatizaciya gidrologicheskih raschetov / A. A. Volchek // Vodohozyajstvennoe stroitel'stvo i ohrana okruzhayushchej sredy: trudy Mezhdunarodnoj nauchno-prakticheskoj konferencii po problemam vodohozyajstvennogo, promyshlennogo i grazhdanskogo stroitel'stva i ekonomiko-social'nyh preobrazovanij $\mathrm{v}$ usloviyah rynochnyh otnoshenij. / Brest. politekhn. institut. - Biberah - Brest Nottingem, 1998. - S. 55-59.

14. Z. Wang, J. Qiu, and F. Li, "Hybrid Models Combining EMD/ EEMD and ARIMA for Long-Term Streamfow Forecasting," Water, vol. 10, no. 7, p. 853, 2018.

15. Norden E. Huang, Samuel S.P. Shen. The Hilbert-Huang transform and its applications // World Scientific Publishing Co. Pte. Ltd. 2005. - $311 \mathrm{~s}$.

16. Peel MC, G.G.S. Pegram, and T.A. McMahon. 2007: Empirical Mode Decomposition: Improvement and application. In International Congress on Modeling and Simulation, edited by Oxley, L. and D. Kulasiri. Modelling and Simulation Society of Australia and New Zealand, December 2007, 2996-3002.

17. Huang N. E., etc. The Empirical Mode Decomposition and The Hilbert Spectrum for Non-linear and Non-stationary Time Series Analysis// Proc. Royal Soc. London, Vol. 454, p. 903-995, 1998.

18. Dolgal', A. S. Primenenie empiricheskoj modovoj dekompozicii pri obrabotke geofizicheskih dannyh / A. S. Dolgal', L. A. Hristenko // Izv. Tomskogo politekhnich. un-ta. Inzhiniring resursov. - 2017. - T. 328. № 1. - S. 100-108.

19. Ajvazyan, S. A. Metody ekonometriki: uchebnik / S. A. Ajvazyan. M. : Infra-M, 2014. - 512 s.

20. Hristenko, L. A. Empiricheskaya modovaya dekompoziciya pri interpretacii dannyh elektrorazvedki / L. A. Hristenko // Geologiya i poleznye iskopaemye Zapadnogo Urala. - Perm', 2019. - № 2(39). - S. 222-226.

Материал поступил в редакцию 19.02.2021 